Pacific Journal of Mathematic 


\title{
GEOMETRIC INTERSECTION FUNCTIONS AND HAMILTONIAN FLOWS ON THE SPACE OF MEASURED FOLIATIONS ON A SURFACE
}

\section{Athanase Papadopoulos}

\begin{abstract}
We define and study some flows on the space of measured foliations on a closed surface, and we relate these flows to the piecewise-linear symplectic geometry of that space, defined by $W$. Thurston.
\end{abstract}

\section{Introduction, statement of results, and notations.}

1.1. The main object of this paper is to define and study some flows on the space of measured foliations on a closed surface (or equivalently on the space of measured geodesic laminations on a closed hyperbolic surface). We interpret these flows in terms of the symplectic geometry of these spaces.

The interest in studying properties of these spaces, classically denoted by $\mathscr{M} \mathscr{F}$ and $\mathscr{M} \mathscr{L}$ respectively, has its origin in Thurston's work on diffeomorphisms of surfaces and their action on Teichmüller space. Up to now, the notions of geodesic lamination and measured foliation have turned out to be essential tools in at least the following three areas:

- deformations of hyperbolic structures on surfaces (see for example the work of Kerckhoff [Ke] and Thurston [Th 2]),

- the study of the mapping class group (see the work of Thurston described in [FLP] or in [Ca]),

- deformations of Kleinian groups, where geodesic laminations interfere via pleated surfaces in hyperbolic 3-manifolds (see the work of Thurston [Th 1]).

Besides these applications, it follows from Thurston's work that the space of measured foliations has a rich geometry and deserves to be studied for itself. (It is understood from now on that the assertions that are made about the measured foliations space are equally valid for the measured geodesic laminations space.) Many features of the geometry of $\mathscr{M} \mathscr{F}$ can be nicely described using the theory of train tracks on surfaces. These objects, introduced by Thurston in [Th 1], can be used to define a 
PL atlas for the space $\mathscr{M} \mathscr{F}$, such that with respect to this atlas, the mapping class group of the surface, acting on $\mathscr{M} \mathscr{F}$, acts piecewise linearly.

This PL structure has been so far utilized in several ways to prove theorems about the mapping class group. Just to give a few examples we mention, besides Thurston's work, work by John McCarthy [McC], Bob Penner [Pe] and the author [Pa 1].

Besides being a natural PL manifold, Thurston has shown that the space $\mathscr{M} \mathscr{F}$ carries in some sense a natural symplectic structure, which we now describe.

Recall that a symplectic structure, in the usual sense, is a closed nondegenerate differential 2-form on a differentiable manifold.

The space of measured foliations does not have a tangent space in the usual sense because the natural change of coordinate transformations are only piecewise smooth. Nevertheless, we can define a symplectic structure on this space in the following sense:

On each local parameters space associated to a maximal train track (cf. definition in $\$ 1.2$ below), there is a non-degenerate closed 2-form which has an invariance property with respect to the coordinate change maps which we now describe: Recall, first, that the domain of each such map $\phi$ is a convex cell of dimension $6 g-6$ and that $\phi$ is piecewise linear. The form is invariant, in the usual sense, when $\phi$ is restricted to the subset of its domain obtained by deleting the set of points in each neighborhood of which $\phi$ is not linear. This set of "defects" is a union of codimension-1 subcells. The invariance property follows from the fact that the symplectic structure behaves well with respect to certain natural operations on the train track (shifts and collapses).

We will recall at the beginning of $\S 4$ the definition of the symplectic form in coordinates.

This symplectic structure on $\mathscr{M} \mathscr{F}$ has been used by W. Floyd in the study of boundary curves of incompressible surfaces in 3-manifolds (see [Fl]).

Using this structure, we obtained in [Pa 4] a quick proof of a theorem which is primarily due to Katok [Ka], which asserts that the number of linearly independent transverse measures for any foliation of a closed surface of genus $g$ is at most $3 g-3$. For another proof, we refer the reader to [Le].

We go on describing how one can make use of this structure:

Recall that a symplectic structure on a differentiable manifold establishes a duality between vectorfields and differential 1-forms. Vectorfields dual to differentials of functions are called hamiltonian vectorfields. 
Even though the space of measured foliations does not possess a tangent bundle in the usual sense, we showed in [Pa 4] that it makes sense to talk about hamiltonian flows which admit in some precise sense tangent vectorfields which are dual to differentials of intersection functions $i(\gamma, \cdot)$, where $\gamma$ is a simple closed curve on the surface. Such a flow is defined on the complement of the codimension-1 submanifold of $\mathscr{M} \mathscr{F}$ defined by the equation $i(\gamma, \cdot)=0$ (this equation characterizes the set of points around which there is no natural coordinate system in which the function $i(\gamma, \cdot)$ is smooth). One can describe the flowline through a point $\mathscr{F}$ in $\mathscr{M}$ satisfying $i(\gamma, \mathscr{F}) \neq 0$ by cutting the surface which is equipped with a foliation representing the class $\mathscr{F}$, along a curve isotopic to $\gamma$ and transverse to the foliation, and regluing after a twist. This is described in detail in [Pa 4], and we shall recall the exact definitions as well as the main constructions in $\$ 4$ below since we will make use of them.

1.2. We proceed in $\S 4$ in the investigation of the symplectic geometry of $\mathscr{M} \mathscr{F}$ in the following ways:

We first remark that if we consider, instead of the intersection function $i(\gamma, \cdot)$, the square of this function, we can also describe its dual flow, with respect to the symplectic structure, and this flow is defined on the whole space $\mathscr{M} \mathscr{F}$. Indeed, the flow which is initially defined on the open set $\mathscr{M} \mathscr{F}-\{i(\gamma, \cdot)=0\}$ is seen to extend by the identity on the set $\{i(\gamma, \cdot)=0\}$.

As a matter of fact, the function $i(\gamma, \cdot)^{2}$, unlike the function $i(\gamma, \cdot)$, is differentiable at each point of the set $\{i(\gamma, \cdot)=0\}$ (since this is the square of a Lipschitz function which takes the value 0 at such points) and the differential at such points is zero. This explains the fact that the dual vectorfield vanishes on this set.

We then generalize this study by associating to each measured foliation a flow defined on $\mathscr{M} \mathscr{F}$. It turns out that the adequate function on $\mathscr{M} \mathscr{F}$ to consider, associated to a measured foliation $\mathscr{G}$, is $\sum_{j=1}^{k} i\left(\mathscr{G}_{j}, \cdot\right)^{2}$, where $\mathscr{G}_{1}, \ldots, \mathscr{G}_{k}$ are the components of $\mathscr{G}$ (to be defined below). This function defines, via the symplectic structure on $\mathscr{M} \mathscr{F}$, a flow on this space which has a qualitative interpretation as a "twist along the measured foliation $\mathscr{G}$ ", which generalizes the discrete twist corresponding to the case where $\mathscr{G}$ is in the class of a simple closed curve.

Of course, the flows are equally well defined on the space $\mathscr{M} \mathscr{F}$ and on the space $\mathscr{M} \mathscr{L}$. But the description as twist deformations on the surface uses measured foliations rather than laminations.

With this in mind, we give in $\$ 2$ necessary and sufficient conditions for two elements of $\mathscr{M} \mathscr{F}$ to admit representatives by transverse measured 
foliations, and in $\$ 3$ we characterize, for a given measured foliation $\mathscr{G}$, the set of points for which there exist train track neighborhoods on which the function $i(\mathscr{G}, \cdot)$ is linear. These sections will be used in $\S 4$ in the proof of the main theorem; they may be of independent interest.

1.3. We now fix some notations and conventions that are used throughtout the rest of the paper. We refer to the book by Fathi, Laudenbach and Poénaru [FLP] and to Chapters 8 and 9 of Thurston's notes [Th 1] for the background material.

$S$ denotes a closed oriented surface of genus $\geq 2$, equipped, when it is necessary, with a hyperbolic metric.

$\mathscr{S}$ is the set of isotopy classes of connected simple closed curves on $S$, non-homotopic to a point.

Recall that $\mathscr{M} \mathscr{F}$ is the set of equivalence classes of measured foliations on $S$ with generalized saddle singularities (three prongs or more), where the equivalence relation is generated by isotopy and Whitehead moves (i.e. collapsing saddle connections), and $\mathscr{M} \mathscr{L}$ is the set of measured geodesic laminations on $S$.

The spaces $\mathscr{M} \mathscr{F}$ and $\mathscr{M} \mathscr{L}$ both inject in the space $R_{+}^{\mathscr{S}}$ of positive functions on $\mathscr{S}$; they have the same image. This allows us to talk about a measured foliation and a measured lamination to be in the same class.

When we will consider geometric measured foliations (i.e. not up to equivalence) it will be convenient for us to distinguish between a total measured foliation (i.e. whose support is $S$ ) and a partial measured foliation (whose support is a subsurface of $S$ ). Remark that with this convention, a geodesic lamination is not a partial foliation since its support has empty interior.

We shall also distinguish between two kinds of partial foliations:

- partial foliations with singularities, which are total measured foliations for a subsurface with boundary, in the usual sense described in [FLP], exposé 11.

- partial foliations with cusps, where the singular points are all on the boundary of the support and are of the type described in Figure 1.

A partial foliation, in either sense, gives a well-defined element of $\mathscr{M} \mathscr{F}$.

A measured geodesic lamination has a finite (and bounded) number of minimal sublaminations, which we will call its components. In a component each half-leaf is dense. The components of a measured lamination are also elements of $\mathscr{M} \mathscr{L}$ and we thus have an intrinsic notion of 


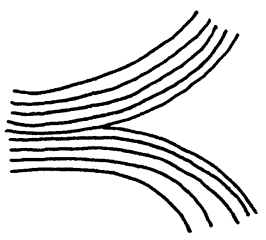

FIGURE 1

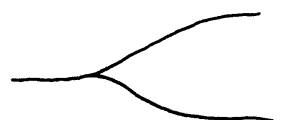

FIGURE 2

components for elements of $\mathscr{M} \mathscr{F}$. We can also recover the components of an element in $\mathscr{M} \mathscr{F}$ by taking a geometric (total or partial) representative and spreading along all cycles of singular leaves. The connected foliated components that we get are either annuli foliated by circles or components in which every leaf is dense. We call them respectively annular or minimal components; they define elements of $\mathscr{M} \mathscr{F}$ which are the components of the equivalence class of the foliation we started with.

Recall that a train track $\tau$ on $S$ is a closed 1-submanifold with switches (Fig. 2) s.t. the components of $S-\tau$ do not include a disk with at most 2 cusps or an annulus with no cusps. A system of weights on $\tau$ is the assignment of a nonnegative number to each branch of $\tau$ s.t. at each switch the total mass on each side is the same.

A train track with weights gives a well-defined element of $\mathscr{M} \mathscr{F}$ (or $\mathscr{M} \mathscr{L}$ ). The set of weights on a given train track $\tau$ defines a convex cell in $\mathscr{M} \mathscr{F}$. When this cell is the closure of an open set in $\mathscr{M} \mathscr{F}$, the train track is said to be maximal. Recall that this holds if and only if all the components of $M-\tau$ are disks with 3 cusps and $\tau$ is recurrent (i.e. $\tau$ carries a system of strictly positive weights). The reader can refer to [Pa 2], Prop. III.1 for a proof of this (otherwise well-known) fact.

We shall be using the following notions of transversality:

Transversality between two total foliations on $S$ is defined in the usual way: the singular sets coincide, at each singular point the number of prongs is the same for each foliation, and in the complement of the singular set the leaves are mutually transverse.

We shall say that a partial foliation with singularities, $\mathscr{G}$, is transverse to a total foliation $\mathscr{F}$ if the singular set of $\mathscr{G}$ is contained in the singular set of $\mathscr{F}$, and in the complement of the singular sets, $\mathscr{G}$ and $\mathscr{F}$ meet transversely. Possible local models at the singularities of $\mathscr{F}$ are given in Figure 3 (there are no non-allowed configurations). The shaded regions indicate the support of $\mathscr{G}$. 


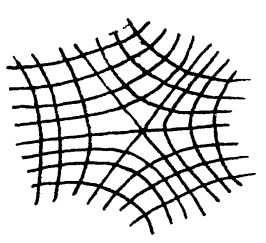

(a)

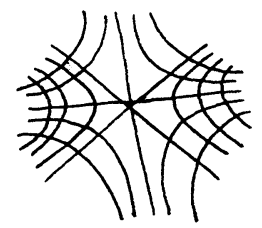

(b)

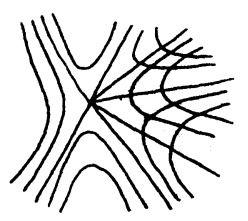

(c)

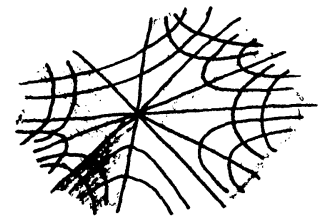

(d)

FIGURE 3

If $\mathscr{G}$ is a partial foliation with cusps and $\mathscr{F}$ is a total foliation, we define $\mathscr{G}$ to be transverse to $\mathscr{F}$ if the support of $\mathscr{G}$ does not intersect the set of singular points of $\mathscr{F}$ and if the leaves meet transversely (transversality makes sense even at the cusps; see Fig. 4).
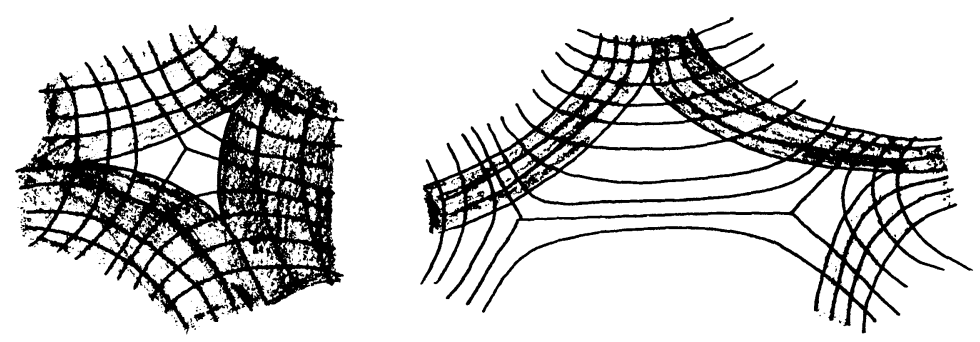

Figure 4

In the same way, a train track $\tau$ is said to be transverse to a total foliation $\mathscr{F}$ if $\tau$ does not meet the set of singular points of $\mathscr{F}$, and is transverse to the leaves.

For the definition and basic properties of the geometric intersection function $i(\cdot, \cdot)$ defined on $\mathscr{M} \mathscr{F} \times \mathscr{M} \mathscr{F}$ we refer to Rees [Re], $\$ 1$.

A last word about notation: $\operatorname{Supp} \mathscr{F}$ will denote the support of $\mathscr{F}$ and $\partial \operatorname{Supp} \mathscr{F}$ the boundary of Supp $\mathscr{F}$.

\section{Transverse foliations.}

Let $\gamma$ and $\lambda$ be 2 measured geodesic laminations on $S$. In this section, we prove the following 2 propositions:

PROPOSITION 1. The following 3 properties are equivalent:

(i) For each $j=1, \ldots, n$, we have $i\left(\gamma_{j}, \lambda\right) \neq 0$, where $\gamma_{1}, \ldots, \gamma_{n}$ are the components of $\gamma$.

(ii) Each half-leaf of $\gamma$ has nonempty transverse intersection with $\lambda$.

(iii) There exists a total measured foliation $\mathscr{L}$, in the class of $\lambda$, and a partial measured foliation with singularities $\mathscr{G}$, in the class of $\gamma$, such that $\mathscr{G}$ 
is transverse to $\mathscr{L}$ (in the sense given in §1.3). We can assume furthermore that the following 2 technical conditions hold: the connected components of $\operatorname{Supp} \mathscr{G}$ are in one-to-one correspondence with the components of $\mathscr{G}$ as a foliation and at each singularity of $\mathscr{L}$ there is (locally) at most one component of $\operatorname{Supp} \mathscr{G}$ (i.e. configurations of the types (b) and (d) in Figure 3 are not allowed).

PROPOSITION 2. The following 4 properties are equivalent:

(iv) There exists a total measured foliation $\mathscr{L}$, in the class of $\lambda$, and a total measured foliation $\mathscr{G}$, in the class of $\gamma$, such that $\mathscr{G}$ is transverse to $\mathscr{L}$.

(v) For every element $C \in \mathscr{S}$, we have $i(C, \gamma)+i(C, \lambda)>0$. Furthermore, no component of $S$-(Supp $\gamma \cup \operatorname{Supp} \lambda)$ has infinite cusps.

(vi) Each component of $S-(\operatorname{Supp} \gamma \cup \operatorname{Supp} \lambda)$ is compact and simply connected.

(vii) For each element $\gamma^{\prime}$ of $\mathscr{M} \mathscr{L}$, we have $i\left(\gamma^{\prime}, \gamma\right)+i\left(\gamma^{\prime}, \lambda\right)>0$.

REMARK. Some of the above implications are straightforward; (ii) $\Rightarrow$ (iii) and (vii) $\Rightarrow$ (iv) use the techniques of train track approximations and some related constructions.

For completeness we provide proofs for all of them.

It will be useful to have in mind the interpretation of the intersection function of 2 transverse measured laminations as the total mass of the 2-dimensional measure on $S$ which is given locally as the product of the 2 transverse measures (cf. Thurston [Th 1], §9.3).

Proof of (i) $\Rightarrow$ (ii). If some half-leaf of $\gamma$ does not cross $\lambda$ transversely, the closure of this half-leaf would be a component of $\gamma$ having zero intersection number with $\lambda$.

Proof of (ii) $\Rightarrow$ (iii). We begin by taking train track approximations of $\gamma$ and $\lambda$ (cf. Thurston [Th 2], §8.9).

Recall that an $\varepsilon$-approximation $\tau_{\varepsilon}(\gamma)$ of $\gamma$ is obtained by first constructing a canonical foliation $\mathscr{F}(\gamma)$, by pieces of horocycles, on the part of $S$ where leaves of $\gamma$ are no more than $\varepsilon$ apart. The train track $\tau_{\varepsilon}(\gamma)$ is a realization of the image of $\gamma$ by the map which collapses each leaf of $\mathscr{F}(\gamma)$ to a point. In the same manner we have $\varepsilon$-train track approximations $\tau_{\varepsilon}(\lambda)$ of $\lambda$.

By compactness of the supports of the laminations, the angle, at a point of intersection, made by a leaf of $\gamma$ and a leaf of $\lambda$, is uniformly 


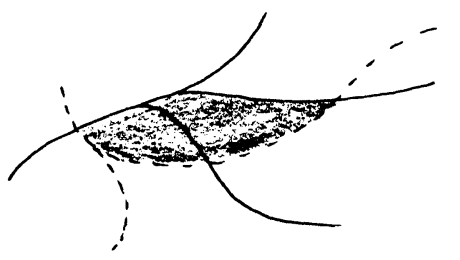

$\tau(\gamma)$ in dotted lines

$\tau(\lambda)$ in fat lines

FIGURE 5

bounded below by a strictly positive number. By taking $\varepsilon$ sufficiently small, we can therefore assume that $\tau_{\varepsilon}(\gamma)$ and $\tau_{\varepsilon}(\lambda)$ are transverse and satisfy the following property: there is no imbedded disk in $S$ whose boundary consists of the union of a smooth arc in $\tau(\gamma)$ and a smooth arc in $\tau(\lambda)$ (see Figure 5). (Remark: it is equivalent, by Euler characteristic, to assume that there is no such disk, with its interior disjoint from $\tau_{\varepsilon}(\gamma)$ and $\left.\tau_{\varepsilon}(\lambda)\right)$.

We now delete the index $\varepsilon$ and denote the train tracks by $\tau(\gamma)$ and $\tau(\lambda)$.

Each of these train tracks is equipped with a system of strictly positive weights, corresponding respectively to the classes in $\mathscr{M} \mathscr{L}$ of the measured laminations $\gamma$ and $\lambda$.

By thickening $\tau(\lambda)$ according to the weights it carries, we get a partial measured foliation with cusps, $\mathscr{L}^{\prime}$, in the same class as $\lambda$, which is transverse to $\tau(\gamma)$ and such that there is no imbedded disk in $S$ whose boundary consists of the union of a smooth arc in $\tau(\gamma)$ and a smooth arc in $\partial \operatorname{Supp} \mathscr{F}^{\prime}$. (We shall call such a disk a bigon.)

The hypothesis (property (ii)) implies that each component of $\tau(\gamma)$ intersects $\mathscr{L}^{\prime}$.

Claim: By performing a finite number of splittings on the train track $\tau(\gamma)$, we can manage so that all of its switches are contained in $\operatorname{Supp} \mathscr{L}^{\prime}$ (keeping the above properties of $\tau(\gamma)$ and $\mathscr{L}^{\prime}$ ).

The operation of splitting a train track is defined in Figure 6, in which $x$ and $y$ denote the weights on the corresponding branches. Cases (a), (b) or (c) in that figure occur respectively if and only if we have $x>y$, $x<y$, or $x=y$.

In this way, each train track obtained from $\tau(\gamma)$ by a finite number of splittings carries a system of strictly positive weights corresponding to the class of $\gamma$.

Imagine now a partial measured foliation with cusps, representing the class of $\gamma$, and obtained by slightly thickening $\tau(\gamma)$. The operation of 

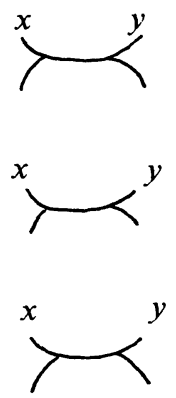
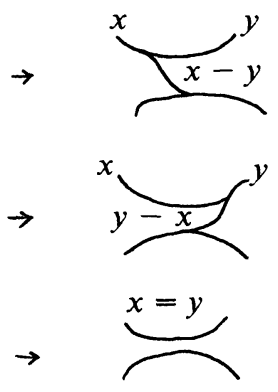

FiguRE 6

splitting the train track with weights corresponds to the operation of opening a cusp, see Figure 7; in other words, we follow the leaf issuing from the cusp. There are 2 (overlapping) cases for such a leaf: either it ends at another cusp (and then we get rid of the 2 cusps by opening the foliation along this leaf (this corresponds to a splitting of type (c)) or such leaf eventually reaches Supp $\mathscr{L}^{\prime}$. Otherwise its closure would give a component of $\gamma$ with zero intersection number with $\lambda$. This implies the claim.

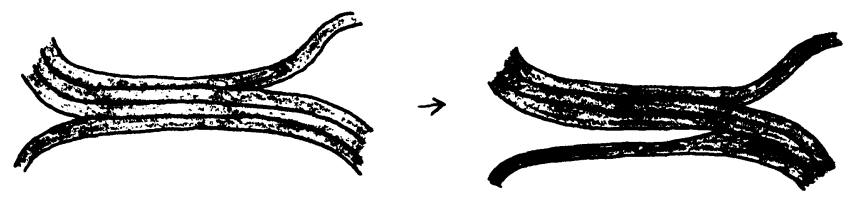

FIGURE 7

We still denote by $\tau(\gamma)$ the new train track obtained.

Each component of $\tau(\gamma)-\operatorname{Supp} \mathscr{L}^{\prime}$ is not a segment whose endpoints lie on $\partial \operatorname{Supp} \mathscr{L}^{\prime}$.

Let $U_{1}, \ldots, U_{l}$ denote the closures of the connected components of $S-\operatorname{Supp} \mathscr{L}^{\prime}$. These are compact surfaces with cusps.

Let $\mathscr{L}^{\prime \prime}$ be the partial measured foliation obtained from $\mathscr{L}^{\prime}$ by pinching each component $U_{i}$ along the segments $U_{i} \cap \tau(\gamma)$ (see the picture in Figure 8). $\tau(\gamma)$ is now contained in Supp $\mathscr{L}^{\prime \prime}$.

The absence of bigons in the figure formed by $\tau(\gamma)$ and $\mathscr{L}^{\prime}$ implies that each component of $S-\operatorname{Supp} \mathscr{L}^{\prime \prime}$, if it is contractible, has at least two cusps. 

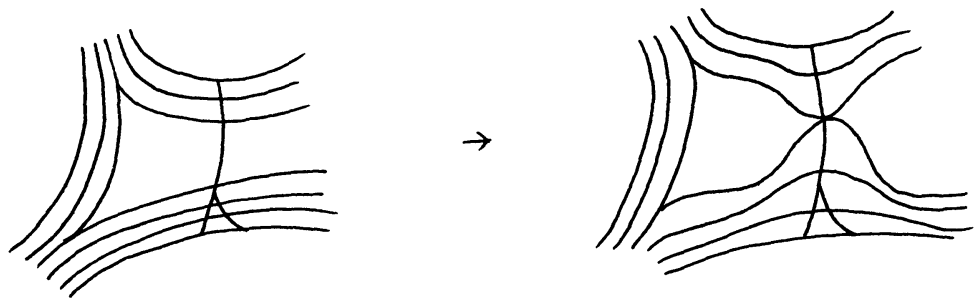

Figure 8

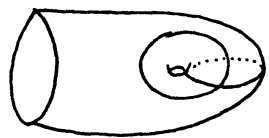

a spine for the surface with boundary

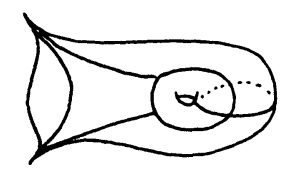

a spine for the surface with boundary with 2 cusps.

\section{FIGURE 9}

Let $\mathscr{L}$ be the total measured foliation obtained from $\mathscr{L}^{\prime \prime}$ by collapsing each component of $S$ - Supp $\mathscr{L}^{\prime \prime}$ on a spine. (Remark: a spine for a compact surface with boundary with cusps is obtained from a spine for a surface with boundary and with no cusps by adding an edge for each cusp. See Figure 9 for an example.)

Summing up, we have a total measured foliation $\mathscr{L}$ representing the class of $\lambda$, transverse to a train track with weights $\tau(\gamma)$, representing the class of $\gamma$.

Let $\mathscr{G}^{\prime}$ be a partial measured foliations with cusps, in the class of $\gamma$, transverse to $\mathscr{L}$, obtained by thickening $\tau(\gamma)$.

By allowing modifications of $\mathscr{L}$ by Whitehead moves, we will replace $\mathscr{G}^{\prime}$ by a partial foliation with singularities, $\mathscr{G}$, in the same class, and transverse to $\mathscr{L}$.

For each cusp of $\mathscr{G}^{\prime}$, we consider the family of arcs induced by leaves of $\mathscr{L}$ on $S-\operatorname{Supp} \mathscr{G}$ " "inside that cusp". The endpoints of each such arc lie on $\partial \operatorname{Supp} \mathscr{G}^{\prime}$. By the "stability lemma" of [FLP], exposé 5 , §II.4, the obstruction to enlarge this family of arcs is the appearance of a singular arc of $\mathscr{L}$ as a limit of arcs in the family (see Figure 10(a)). Let $F$ be such a maximal family.

We modify $\mathscr{L}$ by Whitehead moves so that along this limiting arc, $\mathscr{L}$ has only one singular point (see Figure 10(b)). We then close up the cusp by collapsing each arc in $F$ to a point. The cusp is then replaced by a singularity (see Figure 10(c)).

By performing this operation for each cusp of $\mathscr{G}^{\prime}$, we finally obtain a partial foliation $\mathscr{G}$. (We have to take care of the fact that in performing 


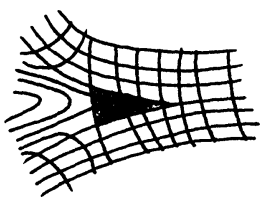

(a)

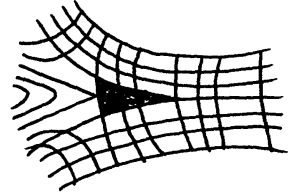

(b)

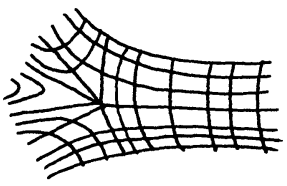

(c)

Figure 10

the operation of closing a cusp, the partial foliation may already pass through the singularity of $\mathscr{L}$ under consideration, but this is straightforward. We obtain local models analogous to those of Figure 3. An example is shown in Figure 11).

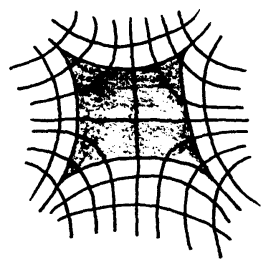

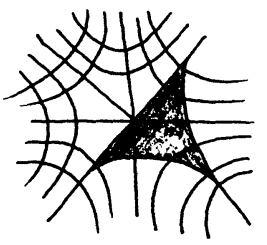

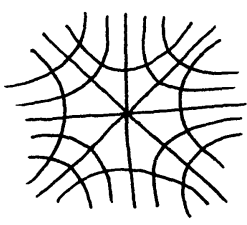

FIGURE 11

Finally, the following remarks take care of the technical condition in property (iii):

By performing enough splitting operations on the train track $\tau(\gamma)$, in the construction above, we can assume that each connected component of the support of $\mathscr{G}^{\prime}$ corresponds to a component of this foliation. We then separate the corners of the partial foliation $\mathscr{G}$ by performing Whitehead moves on $\mathscr{L}$, as it is suggested by the 2 examples given in Figure 12.

Property (iii) is now verified.
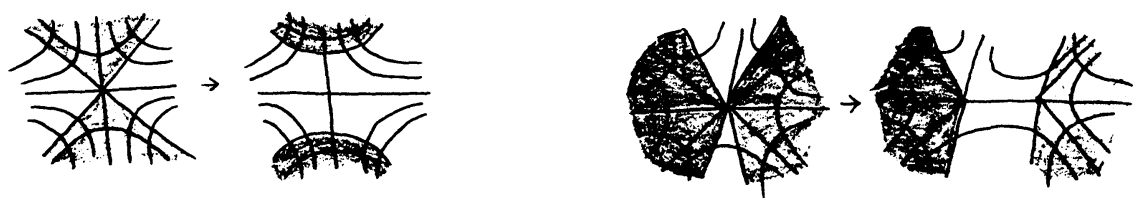

Figure 12

Proof of (iii) $\Rightarrow$ (i). For each component $\gamma_{j}$ of $\gamma$, let $\mathscr{G}_{j}$ be the corresponding component of $\mathscr{G}$. Then $i\left(\gamma_{j}, \lambda\right)=i\left(\mathscr{G}_{j}, \mathscr{L}\right)$ is nonzero since it is the total mass of $\operatorname{Supp} \mathscr{G}_{j}$ with respect to the 2-dimensional measure given locally as the product $\mu_{1} \otimes \mu_{2}$ where $\mu_{1}$ and $\mu_{2}$ are the transverse measures of the foliations $\mathscr{G}$ and $\mathscr{L}$ (this can be seen easily if the component $\mathscr{G}_{j}$ is an annular component and by continuity in the general case). 
Proof of (iv) $\Rightarrow$ (v). If $\mathscr{G}$ and $\mathscr{L}$ are transverse total measured foliations on $S$, they define a singular flat metric, given in the complement of the singularities by $d s^{2}=d \mu_{1}^{2}+d \mu_{2}^{2}$ where $\mu_{1}$ and $\mu_{2}$ are the transverse measures of the foliations $\mathscr{G}$ and $\mathscr{L}$. Each element $C \in \mathscr{S}$ has at least one geodesic representative $C_{0}$ with respect to this metric (see for instance Abikoff [Ab], Ch. 1). A geodesic $C_{0}$ is in particular a quasi-transverse immersion with respect to each of the foliations $\mathscr{G}$ and $\mathscr{L}$ (see [FLP], exposé $5, \S I .7$ for the definition), and the 2 numbers $i(C, \mathscr{G})$ and $i(C, \mathscr{L})$ are therefore given by $\int_{C_{0}} \mu_{1}$ and $\int_{C_{0}} \mu_{2}$ respectively (see [FLP], exposé 6 , $\S \mathrm{V} .4)$. We conclude immediately that the two numbers cannot be simultaneously zero.

To see that no component of $S-(\operatorname{Supp} \gamma \cup \operatorname{Supp} \lambda)$ has infinite cusps, we use the implication (iii) $\Rightarrow$ (ii): $\mathscr{G}$ and $\mathscr{L}$ being both total foliations, every half-leaf of $\gamma$ intersects $\lambda$, and conversely. The picture of the lifts of the laminations to the universal covering of $S$ implies easily that the complementary regions are compact.

Proof of $(\mathrm{v}) \Rightarrow$ (vi). If some component of $S-(\operatorname{Supp} \gamma \cup \operatorname{Supp} \lambda)$ were not simply connected, it would contain a simple closed curve which is non-homotopic (in $S$ ) to a point and which does not meet the boundary of the component; this curve would have zero intersection number with $\gamma$ and $\lambda$.

Proof of $($ vi $) \Rightarrow$ (vii). If a component of $S-(\operatorname{Supp} \lambda \cup \operatorname{Supp} \gamma)$ is compact and simply connected, it cannot contain the support of any element of $\mathscr{M} \mathscr{L}$. We conclude easily that each element of $\mathscr{M} \mathscr{L}$ must have nonempty transverse intersection with $\gamma$ or $\lambda$ (or both), and hence nonzero intersection number with (at least) one of them.

Proof of (vii) $\Rightarrow$ (iv). Notice that (vii) $\Rightarrow$ (i) and therefore (vii) $\Rightarrow$ (iii). Let $\mathscr{G}$ be a partial measured foliation satisfying (iii). For every separatrix of $\mathscr{L}$ which intersects $\operatorname{Supp} \mathscr{G}$, consider the segment between the singularity and the first hitting point on $\partial \operatorname{Supp} \mathscr{G}$. Extend the support of the foliation over all these segments, as indicated in Figure 13, and get a new partial foliation $\mathscr{G}^{\prime \prime}$, in the same class, which has cusps and singularities.
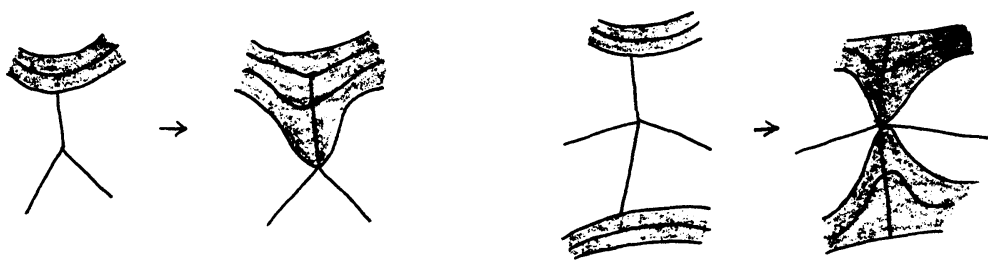

Figure 13 
Remark that each infinite separatrix of $\mathscr{L}$ intersects $\operatorname{Supp} \mathscr{G}$, because otherwise its closure would be a measured foliation having zero intersection number with $\mathscr{G}$ and $\mathscr{L}$.

Consider again the foliation $\mathscr{G}$. Notice that a compact leaf $L$ joining 2 singularities can be disjoint from $\operatorname{Supp} \mathscr{G}$, but is necessarily contained in an imbedded segment $L^{\prime}$ made up of a union of separatrices, with $\partial L^{\prime}$ in $\partial \operatorname{Supp} \mathscr{G}$, as in Figure 14. (To prove this, consider separately the case where $L$ is contained in a closed curve made up of compact separatrices and the case where it is not. In the first case, remark that $\mathscr{L}$ cannot contain a closed union of separatrices which does not meet $\mathscr{G}$, for this would give an element of $\mathscr{S}$ having zero intersection with $\mathscr{G}$ and $\mathscr{L}$. In the second case we are lead to argue as above with infinite separatrices.)

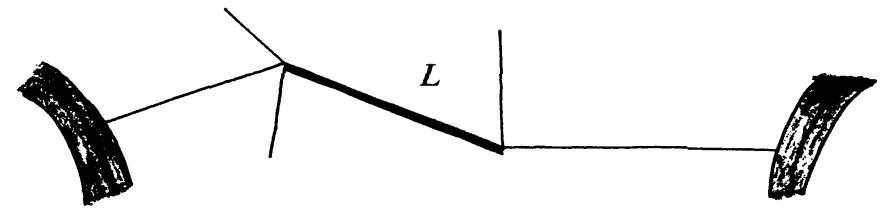

FIGURE 14

By allowing modifications of $\mathscr{L}$ by Whitehead moves, we may therefore assume that at every singularity of $\mathscr{L}$, the germ of each separatrix is contained in $\operatorname{Supp} \mathscr{G}^{\prime \prime}$.

Consider now a component of $M-\operatorname{Supp} \mathscr{G}^{\prime \prime}$. The boundary of such a component has a certain number of singularities, of the type "cusp" or "corner" (see Figure 15(a) and (b)).

The foliation $\mathscr{L}$ induces on this component a foliation transverse to the boundary, with no other singularities than the cusps or corners on its boundary. We conclude by Euler characteristic that such a component is a disk with exactly two singularities on its boundary, and the foliation induced by $\mathscr{F}$ on this disk is a "standard" foliation by segments (see Figure 15(c)). By collapsing each of these segments to a point, we obtain the desired total foliation on the surface.

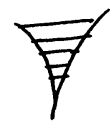

cusp

(a)

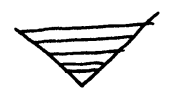

corner

(b)

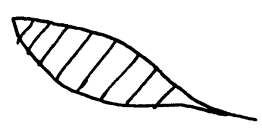

(c) 


\section{The intersection function.}

In this section we prove the following:

Proposition 3. Let $\gamma$ and $\lambda$ be two measured geodesic laminations s.t. for every component $\gamma_{j}$ of $\gamma$, we have $i\left(\gamma_{j}, \lambda\right) \neq$. Then there exists a local coordinate chart given by a maximal train track, defining a neighborhood of $\lambda$, s.t. in these coordinates the intersection function $i(\gamma, \cdot)$ is linear.

REMARKs. (1) The function $i(\gamma, \cdot)$ is equal to $\sum_{j} i\left(\gamma_{j}, \cdot\right)$ where the $\gamma_{j}$ 's are the components of $\gamma$. In any neighborhood of a point $x \in \mathscr{M} \mathscr{L}$ satisfying $i\left(\gamma_{j}, x\right)=0$, the function $i\left(\gamma_{j}, \cdot\right)$ can never be linear since it is a nonnegative function which is not identically zero near any point. From this we can see that the proposition characterizes the set of points of $\mathscr{M} \mathscr{L}$ at the neighborhoods of which we can find local coordinates in which the function $i(\gamma, \cdot)$ is linear. (Using the fact that all the functions are nonnegative, one can see that $i(\gamma, \cdot)$ is linear if and only if $\forall j, i\left(\gamma_{j}, \cdot\right)$ is linear.)

(2) If we take an arbitrary coordinate chart associated to a maximal train track, the proposition implies that the function $i(\gamma, \cdot)$ is piecewise linear in these coordinates, in the neighborhood of each point of the set defined by $i\left(\gamma_{j}, \cdot\right)=0$ for every $j$ (use the fact that the change of coordinates is piecewise linear, cf. Thurston [Th 1], Prop. 9.5.8). The following related result was proved in [Pa 3]: for any element $C \in \mathscr{S}$, the function $i(C, \cdot)$ is piecewise linear on the whole space $\mathscr{M} \mathscr{F}$, in any train track coordinate chart.

Proof of Proposition 3. From Prop. 1 ((i) $\Rightarrow$ (ii)), $\gamma$ and $\lambda$ meet transversely. Furthermore, by the proof of ((ii) $\Rightarrow$ (iii)) of that same proposition, we can find a train track approximation $\tau(\lambda)$ of $\lambda$ satisfying the following properties:

(T1) $\tau(\lambda)$ is transverse to $\gamma$.

(T2) there is no embedded disk in $S$ whose boundary is the union of an arc in a leaf of $\gamma$ and a smooth arc in $\tau(\lambda)$.

By general position we can assume furthermore that

(T3) all the switches of $\tau(\lambda)$ are in the complement of Supp $\gamma$.

REMARK. Generically, the lamination $\lambda$ is complete (i.e. every component of $S-\lambda$ is a disk with 3 (infinite) cusps, cf. [Th 1] Def. 8.7.5). In the case of a complete $\lambda$, each region of $S-\tau(\lambda)$ is the interior of a compact disk with 3 cusps, which implies that $\tau(\lambda)$ is maximal (recall that $\lambda$ gives 
a system of strictly positive weights on $\tau(\lambda)$ ). The considerations that follow deal therefore with the case where the lamination $\lambda$ is not complete.

Consider a region $V$ of $S-\tau(\lambda)$ which is not the interior of a disk with 3 cusps, and let $\bar{V}$ be its natural completion. ( $\bar{V}$ is not the closure of $V$ in $S$; we do not identify pieces on the boundary). The lamination $\gamma$ induces on $\bar{V}$ a lamination of compact support, whose leaves are intervals with endpoints on $\partial \bar{V}$. Call this induced lamination $\gamma_{\mid \bar{V}}$.

Suppose to begin with that there exists a leaft $l$ of $\gamma_{\mid \bar{V}}$ satisfying one (or both) of the following two properties:

(P1) The endpoints of $l$ are not joined on $\partial \bar{V}$ by an arc which has only one cusp (remark that the case of an arc having no cusp is excluded by $\mathrm{T} 2$ ).

(P2) $l$ is not homotopic (relative to its boundary) to an arc (smooth or not) in $\partial \bar{V}$.

We take in $\bar{V}-\gamma_{\mid \bar{V}}$ an arc $l^{\prime}$ satisfying, like $l$, one of the properties (P1) or (P2). (To see that this is possible, recall that transversely the lamination $\lambda$ is either a discrete set of points or a Cantor set.) Without loss of generality, we assume furthermore that the endpoints of $l^{\prime}$ are distinct points on $S$ (even though the endpoints of $l$ can be the same point on $S$ ).

We pinch the train track $\tau(\lambda)$ along the arc $l^{\prime}$, as is indicated in Figure 16, taking care that the support of the operation does not meet the support of $\gamma$. Note that the new train track carries $\lambda$.

Figure 17 is meant to illustrate the case where the endpoints of $l$ are the same point in $S$, i.e. where $l$ is an isolated geodesic.
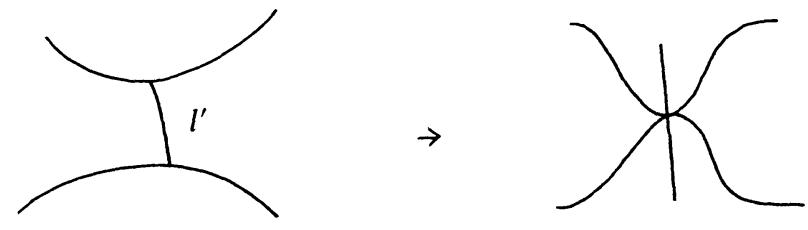

FIGURE 16

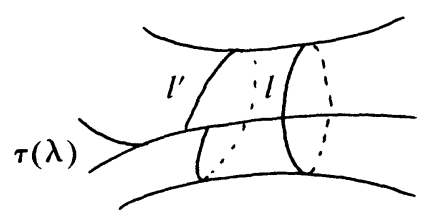

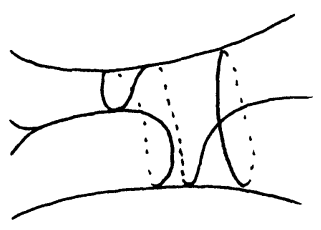




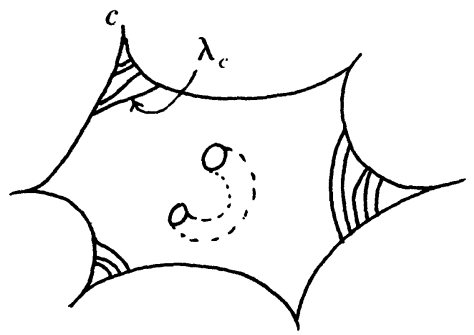

(a)

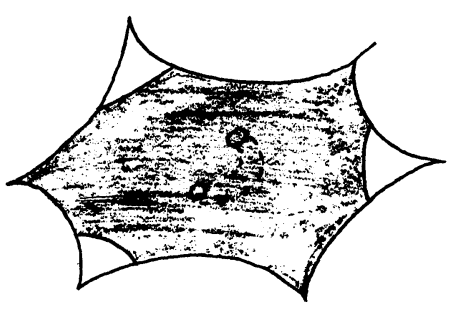

(b)

FigURE 18

By inspecting what are the components of the new train track obtained in this manner, we see that condition (T2) that was satisfied by $\tau(\lambda)$ is also satisfied by the new train track. Conditions (T1) and (T3) are also satisfied by construction.

If there is no leaf of $\gamma_{\mid \bar{V}}$ satisfying one of the 2 properties (P1) and (P2) stated above, each leaf of $\gamma_{\mid \bar{V}}$ bounds a cusp of $\bar{V}$ on one of its sides, as is indicated in Figure 18(a).

By compactness of $\gamma_{\mid \bar{V}}$, for each cusp $c$ of $\bar{V}$ which is bounded by some leaf of $\gamma_{\mid \bar{V}}$, there is an innermost leaf $\lambda_{c}$ having this property (see Figure 18(a)).

Consider the surface $V^{\prime}$ obtained from $\bar{V}$ by chopping all cusps bounded by $\lambda_{c}$ 's (this is the shaded region in Figure 18(b)).

Using the fact that $V$ is not a disk with 3 cusps, we can find a proper arc $l^{\prime \prime}$ in $V^{\prime}$ whose endpoints lie on $\partial V^{\prime}-\left\{\lambda_{c}\right\}$, satisfying with respect to $\bar{V}$ one of the 2 properties (P1) and (P2) stated above for $l$, and such that the endpoints of $l^{\prime \prime}$ are not realized by the same point on $S$.

We pinch $\bar{V}$ along $l^{\prime \prime}$, and verify as in the previous case that the properties (T1) to (T3) that were satisfied by $\tau(\lambda)$ are also satisfied by the train track we get.

In any case, the operation of pinching reduces (in some clear sense upon which we do not insist) the complexity of the component $\bar{V}$, so that after a finite number of operations we end up with a train track $\tau^{*}(\lambda)$ which satisfies the properties (T1) to (T3) that were satisfied by $\tau(\lambda)$ and which is maximal.

Recall that by construction $\lambda$ corresponds to a system of strictly positive weights on $\tau^{*}(\lambda)$, so that this lamination is at the interior of the cell in $\mathscr{M} \mathscr{F}$ parametrized by $\tau^{*}(\lambda)$.

The function $i(\gamma, \cdot)$ is linear in the local coordinates defined by $\tau^{*}(\lambda)$. Indeed, if $a_{1}, \ldots, a_{N}$ are the branches of $\tau$, and $\left(x_{1}, \ldots, x_{N}\right)$ is a system of weights on these branches, corresponding to a measured lamination $\nu$, the quantity $i(\gamma, \nu)$ is equal to $\sum_{i=1}^{N} x_{i}$ meas $\left(a_{i}\right)$, where meas refers 
to the transverse measure of the lamination $\nu$. (This can be first verified for the case where $\nu$ is a weighted simple closed curve, using in an essential way the property that we denoted by (T2). The general case follows by continuity.)

The proof of Proposition 3 is now complete.

\section{Hamiltonian flows on the space of measured foliations.}

4.1. We begin by recalling Thurston's definition of the symplectic structure of $\mathscr{M} \mathscr{F}$.

Let $\tau$ be a maximal train track and $E_{\tau}$ the convex cell of weights associated to $\tau$. Recall that if $\tau$ has $N$ branches, then $E_{\tau}$ sits in $R^{N}$ and is defined by the inequations $x_{i} \geq 0$ for $i=1, \ldots, N$ and the equations of conservation of mass at the switches of $\tau$.

For each point of $E_{\tau}$, we can see the tangent space to $E_{\tau}$ at this point as the subspace of $R^{N}$ generated by this cell, i.e. the set of all (not necessarily positive) weights on the branches of $\tau$, satisfying the equations of conservation of mass at the switches.

If $X=\left(x_{1}, \ldots, x_{N}\right)$ and $Y=\left(y_{1}, \ldots, y_{N}\right)$ are two points in this tangent space, we define their product $\langle X, Y\rangle$ to be $\sum_{s} \frac{1}{2}\left(x_{i_{s}} y_{j_{s}}-x_{j_{s}} y_{i_{s}}\right)$ where the sum is taken over the set of switches of $\tau$, and where for a given switch $s$, the indices $i_{s}$ and $j_{s}$ refer to the branches of $\tau$ that are respectively on the right and on the left as we look inside the cusp. This is well-defined since $S$ is oriented.

It can be easily verified that we get in this way a non-degenerate skew-symmetric product on the tangent space at each point of $E_{\tau}$, that is, a symplectic structure on $E_{\tau}$.

The structure behaves with respect to coordinate changes in a way we have already described in the introduction (\$1.1).

4.2. We now recall the definition of the flow $\left(H_{t}^{\gamma}\right)_{t \in \mathbf{R}}$ that we associated in [Pa 4] to each element $\gamma$ of $\mathscr{S}$.

This flow is defined on the subspace $N(\gamma)$ of $\mathscr{M} \mathscr{F}$ defined by the inequation $i(\gamma, \cdot) \neq 0$ (the letter $N$ in $N(\gamma)$ stands for "Nonzero"). $N(\gamma)$ is the set of points of $\mathscr{M} \mathscr{F}$ for which we can find train track neighborhood coordinates in which the function $i(\gamma, \cdot)$ is smooth. This was proved in [Pa 4] and is a particular case of Proposition 3 in $\$ 3$ above. It was also (easily) proved in [Pa 4] that the subset $Z(\gamma)$ of $\mathscr{M} \mathscr{F}$ defined by the equation $i(\gamma, \cdot)=0$ is a codimension-1 PL-submanifold.

We describe the image $H_{t}^{\gamma}(\mathscr{F})$ under the flow, for each point $\mathscr{F}$ in $N(\gamma)$ 
For each such point $\mathscr{F}$, we can find a representative by a geometric total foliation on $S$ and a representative of the isotopy class $\gamma$ (we call again $\mathscr{F}$ and, respectively, $\gamma$ these representatives) such that $\gamma$ is transverse to $\mathscr{F}$ and does not pass through the singularities. (This was proved in [Pa 4], but is also a consequence of Proposition 1 in $\S 2$ above).

For each $t \in \mathbf{R}$, define a measured foliation $H_{t}^{\gamma}(\mathscr{F})$ by cutting the surface $S$ along $\gamma$, and regluing back the two boundary components of the resulting surface after a twist of strength $t$. The amount of twisting is measured via the measure induced on $\gamma$ by the transverse measure of the foliation $\mathscr{F}$, and the sense of the twist (left or right) is determined by the sign of $t$ (respectively positive or negative). In Figure 19, a left-hand twist is represented.

That the equivalence class of the measured foliation $H_{t}^{\gamma}(\mathscr{F})$ is independent of the particular representatives $\mathscr{F}$ and $\gamma$ in their classes, was proved in [ $\mathbf{P a} 4]$ and is also a consequence of a more general fact that we will prove below (Lemma 6).

This defines a flow $\left(H_{t}^{\gamma}\right)_{t \in \mathbf{R}}$ on $N(\gamma)$.

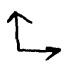

orientation of $S$

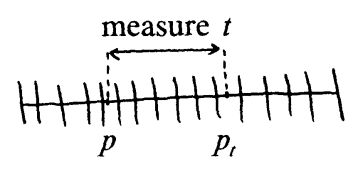

$p_{t}$

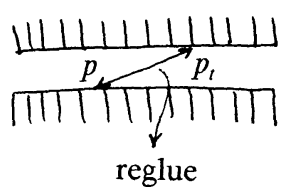

FIGURE 19

4.3. The following theorem was proved in [Pa 4]:

THEOREM 4. The flow $\left(H_{t}^{\gamma}\right)$ has a tangent vectorfield $h^{\gamma}$ which is the dual of the differential of the function $i(\gamma, \cdot)$ restricted to the open set $N(\gamma)$ with respect to the symplectic structure $\langle$,$\rangle restricted to this set. In other$ words, we have at each point $\mathscr{F} \in N(\gamma)$ :

$$
\left\langle h^{\gamma}(\mathscr{F}), \cdot\right\rangle=-(\operatorname{di}(\gamma, \cdot)(\mathscr{F}))(\cdot) .
$$

Remark. Again we insist upon the fact that $\mathscr{M} \mathscr{F}$ being not a smooth, but a PL manifold, this theorem should be interpreted in the following way: we can find in the neighborhood of each point of $N(\gamma)$ a coordinate chart associated to a maximal train track, in which the function $i(\gamma, \cdot)$ is smooth, and in which the flow is smooth and admits a tangent field which is the dual, with respect to the symplectic product $\langle$,$\rangle , to the differential$ of the function. In fact that function $i(\gamma, \cdot)$ will be linear in these special coordinates and the vectorfield will be constant. 
The general picture (i.e. in an arbitrary coordinate chart associated to a maximal train track) is that of a continuous flow admitting a piecewise constant tangent vectorfield.

We recall the main point of the proof of Theorem 4 that we gave in [Pa 4] since we will make use of it.

Sketch of proof of Theorem 4. For any point $\mathscr{F}$ in $N(\gamma)$, we find a maximal train track $\tau$ such that $\mathscr{F}$ corresponds to a system of strictly positive weights on $\tau$ and such that $\gamma$ is represented by a curve imbedded in $\tau$ and meeting exactly two switches of $\tau$, as in one of the Figures 20(a) or (b) (these figures are isomorphic, but they are not the same for our purposes; we are making use of the orientation of $S$ ).
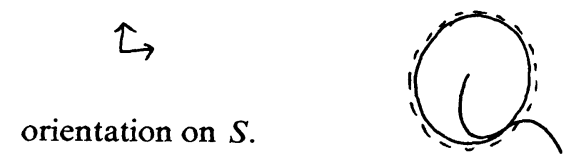

(a)

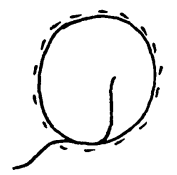

( $\gamma$ in dotted line)

(b)

FIGURE 20

Note that for a given measured foliation $\mathscr{F}$ carried by $\tau$, the 2 weights it assigns to the 2 branches bordering on $\gamma$ are the same, and are equal to $i(\gamma, \mathscr{F})$.

Each of Figures 20(a) and (b) may be described by saying that the branches of $\tau$ bordering on $\gamma$ arrive on this curve respectively "from the left" and "from the right". In each case, half of the flowline passing through the point $\mathscr{F}$ is contained in the subspace of $\mathscr{M} \mathscr{F}$ parametrized by $\left(\left\{H_{t}^{\gamma}(\mathscr{F}), t \leq 0\right\}\right.$ in case (a) and $\left\{H_{t}^{\gamma}(\mathscr{F}), t \geq 0\right\}$ in case (b)).

Imagine now a natural fibered neighborhood $V$ of $\tau$ (see Figure 21(a)) (the fibers are usually called the ties of the train track), and represent $\mathscr{F}$ by a geometric measured foliation $\mathscr{F}(\tau)$ whose support is $V$ and which is transverse to the ties. The weight attributed by $\mathscr{F}$ to each edge of $\tau$ is equal to the total measure of any tie corresponding to that edge, with respect to the transverse measure of $\mathscr{F}(\tau)$.

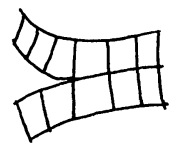

(a)

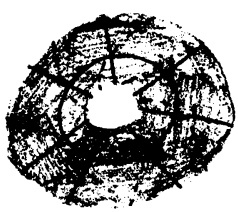

(b) $\gamma^{*}$ is the central curve $\mathscr{F}(\tau)$ in dotted lines the ties in fat lines 
The isotopy class of $\gamma$ can be represented by a curve $\gamma^{*}$ which is transverse to $\mathscr{F}$ and to the ties, and intersects in exactly one point each of the ties corresponding to the 2 branches of $\tau$ traced by $\gamma$, and only these.

From this picture we can see that the twist deformation along $\gamma^{*}$ affects only the measure of the ties that $\gamma^{*}$ crosses. In other words, it only affects the weights on the branches of $\tau$ that are traced by $\gamma$. In view of the definition of the strength of the twist (recall that at time $t$, the total amount of the twist is equal to $t$, with respect to the measure induced on the curve $\gamma^{*}$ ), it can be seen that the flowline passing through the point $\mathscr{F}$ that we are considering has a tangent vectorfield at each point, whose components, in the coordinate system associated to $\tau$, are 1 on the two branches traced by $\gamma$ and 0 elsewhere.

The formula is then immediately seen to be true, using the explicit form of the symplectic product.

4.4. We now associate to every element $\gamma$ in the set $\mathscr{S}$ a new flow, $\left(F_{t}^{\gamma}\right)_{t \in \mathbf{R}}$ defined on $\mathscr{M} \mathscr{F}$.

The flow is first defined on the open set $N(\gamma)$ in much the same way as the flow $\left(H_{t}^{\gamma}\right)$ defined above; the flowlines are the same but the amount of twisting is different. For each $\mathscr{F} \in N(\gamma)$, the flow through that point is defined by cutting the surface along a curve in the class $\gamma$, transverse to a foliation in the class $\mathscr{F}$, and regluing so that at time $t$, the amount of twisting (measured on the curve, again with respect to the transverse measure induced by the foliation) is equal to the product $i(\mathscr{F}, \gamma) \cdot t$.

The sign convention is the same as that for the flow $H_{t}^{\gamma}$.

Remark that the action of the time-one map $F_{1}^{\gamma}$ is that of a Dehn twist along $\gamma$.

The following theorem holds:

THEOREM 5. The flow $\left(F_{t}^{\gamma}\right)_{t \in \mathbf{R}}$ that we have defined on $N(\gamma)$ has a continuous extension to the whole space $\mathscr{M} \mathscr{F}$. It possesses a tangent vectorfield $f^{\gamma}$ which is (up to a constant multiple) the dual of the differential of the function $\mathscr{I}$ defined on $\mathscr{M} \mathscr{F}$ by $\mathscr{I}(\mathscr{F})=i(\gamma, \mathscr{F})^{2}$, with respect to the symplectic structure $\langle\cdot, \cdot\rangle$ on $\mathscr{M} \mathscr{F}$.

The exact formula is, for every $\mathscr{F} \in \mathscr{M} \mathscr{F}$ :

$$
\left\langle f^{\gamma}(\mathscr{F}), \cdot\right\rangle=-\frac{1}{2} d \mathscr{I}(\mathscr{F})(\cdot) \text {. }
$$

REMARK. The same remark than the one following the statement of Theorem 4 holds here. The flow $F_{t}^{\gamma}$ admits a tangent vectorfield in the same sense as the flow $H_{t}^{\gamma}$ does. The general picture for $f^{\gamma}$ is that of a 
piecewise linear vectorfield, in the same way as $H^{\gamma}$ was a piecewise constant vectorfield.

Proof of Theorem 5. One can see the continuity either directly from the definition (since as $\mathscr{F}$ approaches $Z(\gamma)$, the quantity $i(\gamma, \mathscr{F})$ tends to zero), or else from the description-in-coordinates that we shall discuss now.

For the proof of the duality formula, we investigate separately the situations near the points in $N(\gamma)$ and near the points in the complementary set $Z(\gamma)$.

For a point $\mathscr{F}$ in $N(\gamma)$, we use the same train track coordinate system that was used in the proof of the duality formula of Theorem 4.

Referring to Figure 21, we denote by $x$ the weight associated by $\mathscr{F}$ to the branches of $\tau$ bordering on $\gamma$. The quantity $\mathscr{I}(\mathscr{F})$ is then equal to $x^{2}$, and the differential $d \mathscr{I}(\mathscr{F})(\cdot)$ is equal to the following 1-form defined on the tangent space at $\mathscr{F}$ to the cell associated to $\tau$ : If $Y=\left(y_{1}, \ldots, y_{N}\right)$ is a vector in this space (that is, a system of nonnecessarily nonnegative weights on $\tau$ satisfying the switch conditions), we have $d \mathscr{I}(\mathscr{F})(Y)=$ $2 x \cdot y_{e}$, where $y_{e}$ is the coordinate of $Y$ on any of the 2 branches bordering on $\gamma$.

Again, as in the case of the flow $H_{t}^{\gamma}$, half of the flowline passing through $\mathscr{F}$ (that is, $\left\{F_{t}^{\gamma}, t \leq 0\right\}$ or $\left\{F_{t}^{\gamma}, t \geq 0\right\}$ ) is contained in the subset of $\mathscr{M} \mathscr{F}$ parametrized by this chart, depending on whether we are in the case of Figure 20(a) or 20(b).

In any case, the tangent vectorfield to the deformation is constant on this half-flowline, in these coordinates, and has the following components: $i(\gamma, \mathscr{F})$ on the two branches of $\tau$ traced on $\tau$ by $\gamma$, and 0 elsewhere. The proof of this fact is the same as that of the corresponding fact for the flow $H_{t}^{\gamma}$; it amounts to seeing that under the effect of the twist, the weights on the branches of $\tau$ are unchanged except for the 2 branches traced by $\gamma$, for which the rate of variation of the weight is exactly $i(\gamma, \mathscr{F})$.

Recall that $i(\gamma, \mathscr{F})=x$.

By referring to the explicit formula for the symplectic product, we find that for $Y$ as above, an element of the tangent space at $\mathscr{F}$, we have in the coordinates associated to $\tau:\left\langle f^{\gamma}(\mathscr{F}), Y\right\rangle=-x \cdot y_{e}$ where $y_{e}$ has the same meaning as above.

The duality formula is now verified for $\mathscr{F} \in N(\gamma)$.

For a point in $Z(\gamma)$, in view of what we said at the beginning of the proof of the theorem, we must show that the differential of $\mathscr{I}$ vanishes at this point. 
This is easily verified since $\mathscr{I}$ is the square of the Lipschitz function $i(\gamma, \cdot)$ which takes the value 0 at such a point. This completes the proof.

Remarks (1) The function $i(\gamma, \cdot)$ is Lipschitz in any train track coordinate chart since it is piecewise linear (see [Pa 4]); Mary Rees proves in [Re] that for any element $\mathscr{F}$ in $\mathscr{M} \mathscr{F}$, the function $i(\mathscr{F}, \cdot)$ is Lipschitz (with respect to the coordinate charts associated to pants decompositions, but these are related to train track coordinate charts by piecewise linear mappings, so the result holds also in the coordinates we are considering).

(2) The function $i(\gamma, \cdot)$ is not differentiable near a point in $Z(\gamma)$ in any natural coordinate system, since if it were, it would have to be linear near this point and we have already remarked in $\$ 3$ that this is impossible. The situation that we have here should be compared with the trivial fact that the function $x \mapsto|x|$ defined on $\mathbf{R}$ is not differentiable at 0 , whereas its square is.

(3) Another advantage of the flow $F_{t}^{\gamma}$ is that it preserves rays in $\mathscr{M} \mathscr{F}$, whereas $H_{t}^{\gamma}$ does not. The induced flow on $\mathscr{P} \mathscr{M} \mathscr{F}$ interpolates the action of the Dehn twist along $\gamma$ on this space.

4.5. We want to associate a flow $\left(F_{t}^{\mathscr{F}}\right)_{t \in \mathbf{R}}$ to an arbitrary element $\mathscr{F}$ in $\mathscr{M} \mathscr{F}$, in a way that generalizes the flows $F_{t}^{\gamma}$ defined for elements $\gamma$ in $\mathscr{S}$.

We will need the following

LEMMA 6. Let $\mathscr{F}_{1}$ and $\mathscr{F}_{2}$ be 2 equivalent total measured foliations on $S$, and let $\mathscr{G}_{1}$ and $\mathscr{G}_{2}$ be (simultaneously) either:

1 -equivalent total measured foliations transverse respectively to $\mathscr{F}_{1}$ and $\mathscr{F}_{2}$.

2-equivalent partial measured foliations with singularities, transverse respectively to $\mathscr{F}_{1}$ and $\mathscr{F}_{2}$, and satisfying the following 2 technical conditions:

-For $i=1$ and 2, the connected components of $\operatorname{Supp} \mathscr{G}_{i}$ are in one-to-one correspondence with the components of $\mathscr{G}_{i}$ as a foliation.

-For $i=1$ and 2, each singularity of $\mathscr{F}_{i}$ meets, locally, at most one component of $\operatorname{Supp}_{i}$.

( Recall that these conditions appear in property (iii) of Proposition 1.)

Then we can get $\mathscr{F}_{1}$ from $\mathscr{F}_{2}$ by using operations of the following types

-isotopy

- Whitehead moves which involve only separatrices that are contained in the closure of the complement of $\mathscr{G}_{i}$. 
Proof If $\mathscr{G}_{1}$ and $\mathscr{G}_{2}$ are total foliations, then this is given by Proposition 2.7 of Masur [Ma], which asserts that in this case there is an isotopy of the surface which takes $\mathscr{F}_{1}$ to $\mathscr{F}_{2}$ and $\mathscr{G}_{1}$ to $\mathscr{G}_{2}$.

Suppose now that $\mathscr{G}_{1}$ and $\mathscr{G}_{2}$ are not total foliations. By allowing Whitehead moves on $\mathscr{F}_{1}$ and $\mathscr{F}_{2}$ that do not involve separatrices of $\mathscr{F}_{1}$ (resp. $\mathscr{F}_{2}$ ) passing through $\operatorname{Supp} \mathscr{G}_{1}$ (resp. Supp $\mathscr{G}_{2}$ ), we shall construct 2 partial foliations $\mathscr{G}_{1}^{\prime}$ and $\mathscr{G}_{2}^{\prime}$ that are in the same equivalence class, whose support is disjoint respectively from $\operatorname{Supp} \mathscr{F}_{1}$ and $\operatorname{Supp} \mathscr{F}_{2}$, and such that the following 3 properties hold for $i=1$ and 2:

- the partial measured foliation $\mathscr{G}_{i} \amalg \mathscr{G}_{i}^{\prime}$ satisfies all the properties of $\mathscr{G}_{1}$ that are listed in the statement of the lemma.

- Every infinite separatrix of $\mathscr{F}_{i}$ has nonvoid transverse intersection with $\operatorname{Supp} \mathscr{G}_{i} \cup \operatorname{Supp} \mathscr{G}_{i}^{\prime}$.

- Every cycle of finite separatrices of $\mathscr{F}_{i}$ has nonvoid transverse intersection with $\operatorname{Supp} \mathscr{G}_{i} \cup \operatorname{Supp} \mathscr{G}_{i}^{\prime}$.

(Note that $\mathscr{G}_{1}^{\prime}$ and $\mathscr{G}_{2}^{\prime}$ may be the empty foliation.)

The foliations $\mathscr{G}_{1}^{\prime}$ and $\mathscr{G}_{2}^{\prime}$ will be constructed by thickening some simple closed curves on $S$, that we now construct.

Consider an infinite separatrix $L_{1}$ of $F_{1}$ that does not cross Supp $\mathscr{G}_{1}$. The closure of this separatrix is a compact subsurface $S_{1}$ of $S$ with negative Euler characteristic, whose interior is disjoint from $\operatorname{Supp} \mathscr{G}_{1}$. By performing Whitehead moves on $F_{1}$ which do not involve separatrices that cross $\mathscr{G}_{1}$, we can furthermore assume that the boundary of $S_{1}$ does not have corners (recall the construction in Figure 12).

Let $C_{1}$ be a simple closed curve in the interior of $S_{1}$, that is transverse to the foliation induced by $\mathscr{F}_{1}$ on this surface, and that does not pass through a singularity. (In particular, $C_{1}$ is not homotopic to a point on $S$.)

To the separatrix $L_{1}$ there corresponds in a precise way a separatrix $L_{2}$ of $\mathscr{F}_{2}$ (recall that Whitehead moves do not affect infinite separatrices), and the closure of $L_{2}$ is a subsurface, which we desingularize as above (using the operations of Figure 12), and which we call $S_{2}$. As foliated surfaces, $S_{1}$ and $S_{2}$ are equivalent, and by allowing Whitehead moves on the foliation on $S_{2}$ induced by $\mathscr{F}_{2}$, we can find a curve $C_{2}$ which is isotopic to $C_{1}$ on $S$, which is contained in the interior of $S_{2}$, and which is transverse to $\mathscr{F}_{2}$ without passing through singular points (remark that the theory of existence of transverse curves to a measured foliation works for surfaces with boundary in exactly the same way as for closed surfaces, studied in [FLP], exposé 5, §II.6. It only involves simplification of Whitney disks).

By thickening the curves $C_{1}$ and $C_{2}$, we obtain 2 foliated annuli, 
equivalent as measured foliations of $S$ (provided we choose the same total transverse measure), and contained in the complement of $\mathscr{G}_{1}$ and $\mathscr{G}_{2}$.

By iterating this process finitely many times, we end up with a pair of foliations satisfying the first 2 of the 3 properties that we required from $\mathscr{G}_{l} \amalg \mathscr{G}_{\imath}^{\prime}$.

Let $L_{1}$ be now a simple closed cycle of separatrices of $\mathscr{F}_{1}$ that does not intersect transversely the partial foliation (which we denote again by $\mathscr{G}_{1}$ ). Consider the subsurface $S_{1}$ of $S$ defined as the connected component of $S-\operatorname{Supp} \mathscr{G}_{1}$ that contains $L_{1} . S_{1}$ has as above, negative Euler characteristic and is equipped with a foliation, induced by $\mathscr{F}_{1}$, which is transverse to the boundary.

The boundary components of $S_{1}$ can be either closed leaves of $\mathscr{G}_{1}$ or cycles of compact separatrices of $\mathscr{G}_{1}$, and $L_{1}$ is not isotopic to a boundary component of $S_{1}$ since these curves have nonzero intersection number with $\mathscr{F}_{1}$.

By allowing Whitehead moves of $\mathscr{F}_{1}$ involving only separatrices contained in $S_{1}$, we can find a simple closed curve $C_{1}$ in the interior of $S_{1}$, which is transverse to $\mathscr{F}_{1}$, which has nonzero intersection number with $L_{1}$, and which does not pass through singular points.

To the simple closed cycle $L_{1}$ of $\mathscr{F}_{1}$ there corresponds exactly one simple closed cycle $L_{2}$ of $\mathscr{F}_{2}$ in the same isotopy class, and to the surface $S_{1}$ there corresponds exactly one component $S_{2}$ of $S-\operatorname{Supp} \mathscr{G}_{2}$, that contains $L_{2}$. This uses the fact that the components of $\operatorname{Supp} \mathscr{G}_{1}$ and $\operatorname{Supp} \mathscr{G}_{2}$ are in one-to-one correspondence with the components of $\mathscr{G}_{1}$ and $\mathscr{G}_{2}$ as foliations. $S_{1}$ and $S_{2}$ are isotopic subsurfaces of $S$.

Again we can find, by performing Whitehead moves on $\mathscr{F}_{2}$ involving only separatrices contained in the surface $S_{2}$, a simple closed curve $C_{2}$ isotopic to $C_{1}$, transverse to $\mathscr{F}_{2}$, which crosses $L_{2}$ and does not pass through a singular point.

By thickening $C_{1}$ and $C_{2}$, and repeating this construction as many times as is needed, we end up with the required foliations $\mathscr{G}_{1} \amalg \mathscr{G}_{1}^{\prime}$ and $\mathscr{G}_{2} \amalg \mathscr{G}_{2}^{\prime}$.

By the proof of (vii) $\Rightarrow$ (iv) in $\$ 2$, we know that we can thicken $\mathscr{G}_{1} \amalg \mathscr{G}_{1}^{\prime}$ and $\mathscr{G}_{2} \amalg \mathscr{G}_{2}^{\prime}$ to get total measured foliations transverse respectively to $\mathscr{F}_{1}$ and $\mathscr{F}_{2}$, by allowing Whitehead moves on $\mathscr{F}_{1}$ and $\mathscr{F}_{2}$ that involve only separatrices in the complement of $\operatorname{Supp}\left(\mathscr{G}_{1} \amalg \mathscr{G}_{1}^{\prime}\right)$ and $\operatorname{Supp}\left(\mathscr{G}_{2} \amalg \mathscr{G}_{2}^{\prime}\right)$ respectively.

We are now reduced to the case where $\mathscr{G}_{1}$ and $\mathscr{G}_{2}$ are total foliations that was studied at the beginning of the proof.

The proof of Lemma 6 is now complete. 
4.6. We now associate to an arbitrary element $\mathscr{G} \in \mathscr{M} \mathscr{F}$, a flow $\left(F_{t}^{\mathscr{G}}\right)_{t \in \mathbf{R}}$ on the space $\mathscr{M} \mathscr{F}$.

We begin by defining the flow on the subspace $N(\mathscr{G})$ of $\mathscr{M} \mathscr{F}$ defined by $i\left(\mathscr{G}_{j}, \cdot\right) \neq 0$ for every $j=1, \ldots, k$, when $\mathscr{G}_{1}, \ldots, \mathscr{G}_{k}$ are the components of $\mathscr{G}$.

Let $\mathscr{F}$ be an element of $N(\mathscr{G})$ and let us describe the flowline through $\mathscr{F}$.

Using Proposition 1, we represent $\mathscr{F}$ and $\mathscr{G}$ by 2 geometric measured foliations that we will also denote by $\mathscr{F}$ and $\mathscr{G}$, satisfying property (iii) of this proposition.

Let $\mathscr{G}_{1}, \ldots, \mathscr{G}_{k}$ denote also the components of the geometric foliation $\mathscr{G}$.

Supp $\mathscr{G}$ is equipped with a pair of transverse foliations, a "horizontal" one induced by $\mathscr{G}$, and a "vertical" one induced by $\mathscr{F}$. This defines a geometric structure on $\operatorname{Supp} \mathscr{G}-\{$ singularities $\}$ in which one can talk of angles, of straight lines, of horizontal lines and of vertical lines.

For each $t \in \mathbf{R}$, we define $F_{t}^{\mathscr{G}}(\mathscr{F})$ to be the geometric measured foliation on $S$ which coincides with $\mathscr{F}$ in the complement of $\operatorname{Supp} \mathscr{G}$, and which is given, for each $i=1, \ldots, k$, on $\operatorname{Supp} \mathscr{G}_{i}$ by straight lines making with the vertical lines an angle $\alpha$ defined by $\tan \alpha=t \cdot i\left(\mathscr{G}_{i}, \mathscr{F}\right)$. Depending on the sign of $t$, the angle is measured either in the clockwise or counterclockwise direction. This makes use of the orientation on $S$. The singular points of $F_{t}^{\mathscr{G}}(\mathscr{F})$ are the same as those of $\mathscr{F}$.

It follows from Lemma 6 that the equivalence class in $\mathscr{M} \mathscr{F}$ of the foliation $F_{t}^{\mathscr{G}}(\mathscr{F})$ depends only on the classes of $\mathscr{G}$ and $\mathscr{F}$ and not on the particular representatives that were chosen for the definition.

The flow which has been up to now defined on $N(\mathscr{G})$, extends on the whole space $\mathscr{M} \mathscr{F}$. Indeed, as the intersection numbers of a sequence of elements in $N(\mathscr{G})$, with $\mathscr{G}_{i}$, approach zero, the angle of bending in the geometric definition of the flow tends to zero, so that the flow extends on $\mathscr{M} \mathscr{F}$ by defining the image $F_{t}^{\mathscr{G}}(\mathscr{F})$ of an arbitrary element $\mathscr{F} \in \mathscr{M} \mathscr{F}$ to be its time- $t$ image under the flow associated to the foliation obtained from $\mathscr{G}$ by deleting the components $\mathscr{G}_{j}$ which satisfy $i\left(\mathscr{G}_{j}, \mathscr{F}\right)=0$. In particular, the flow is the identity on the set of points defined by $i\left(\mathscr{G}_{j}, \cdot\right)=0 \forall j=1, \ldots, k$ or equivalently by the single equation $i(\mathscr{G}, \cdot)=$ 0 .

Remark also that it follows from the definition that the flow $F^{\mathscr{G}}$ preserves rays in $\mathscr{M} \mathscr{F}$ and therefore defines a flow on $\mathscr{P} \mathscr{M} \mathscr{F}$.

For any $\mathscr{G}$, the flow $F^{\mathscr{G}}$ is continuous. The continuity comes from a general principle which says that when 2 geometric measured foliations 
have the same singular set and are close together geometrically (i.e. in the topology of the set of unoriented directions on $S$ ), their classes in $\mathscr{M} \mathscr{F}$ are close together in the topology of $\mathscr{M} \mathscr{F}$. This is described in [FLP], exposé $6, \S \mathrm{V}$, and uses the existence (for each geometric measured foliation) of a quasi-transverse immersed curve in each class in $\mathscr{S}$, realizing the minimum of intersection number in their class.

If $\gamma$ is an element of $\mathscr{S}$, the flow $F_{t}^{\gamma}$ that we have just defined coincides with the flow that we defined in $\$ 4.4$.

To see this, we remark by going back to Figure 22(b) and using the same notations as there, that the effect of the twist of strength $t \cdot i(\gamma, \mathscr{F})$ along the curve $\gamma^{*}$ is the same as the effect of taking a foliated annulus of width 1 , transverse to $\mathscr{F}(\tau)$, and bending the foliation induced by $\mathscr{F}(\tau)$ on the annulus by an angle whose tangent is $t \cdot i(\gamma, \mathscr{F})$, measured in the flat structure induced on the annulus by the 2 transverse foliations.

We saw (Theorem 5) that the flow $F^{\gamma}$ on $\mathscr{M} \mathscr{F}$ associated to an element $\gamma \in \mathscr{S}$ is the hamiltonian flow of the function $i(\gamma, \cdot)^{2}$ with respect to the symplectic structure of that space. It can be easily verified that the same thing holds if we take, instead of an element $\gamma \in \mathscr{S}$, an element of the form $\lambda \gamma$ where $\lambda \in \mathbf{R}$ and $\gamma \in \mathscr{S}$. For this we compute in the same train track coordinates used in the proof of Theorem 5. Using the same notations as there, the function $i(\lambda \gamma, \cdot)^{2}$ is given by $\lambda^{2} x^{2}$, the hamiltonian vectorfield is the one of the function $\lambda^{2} \cdot i(\gamma, \cdot)^{2}$. We represent $\lambda \cdot \gamma$ by a foliated annulus, transverse to $\mathscr{F}(\tau)$ and whose total width is $\lambda$. The hamiltonian flow acts by bending the vertical foliation by an angle whose tangent is $t \lambda^{2} x / \lambda=t \lambda x$, which is therefore the action of the flow $F_{t}^{\lambda \gamma}$.

Remark now that every component $\mathscr{G}_{j}$ of an element $\mathscr{G} \in \mathscr{M} \mathscr{F}$ can be approximated by a sequence $\lambda_{n} \gamma_{n}$ of weighted elements of $\mathscr{S}$ and the convergence of $\lambda_{n} \gamma_{n}$ to $\mathscr{G}_{j}$ is a necessary and sufficient condition for the functions $i\left(\lambda_{n} \gamma_{n}, \cdot\right)^{2}$ to converge to the function $i\left(\mathscr{G}_{j}, \cdot\right)^{2}$.

Summing up, we have, for each $\mathscr{G} \in \mathscr{M} \mathscr{F}$, a function on $\mathscr{M} \mathscr{F}$ defined by $\sum_{j} i\left(\mathscr{G}_{j}, \cdot\right)^{2}$, where the $\mathscr{G}_{j}$ 's are the component of $\mathscr{G}$. Each function $i\left(\mathscr{G}_{j}, \cdot\right)^{2}$ is a limit of functions $i\left(\lambda_{n} \gamma_{n}, \cdot\right)^{2}$ whose hamiltonian flow on $\mathscr{M} \mathscr{F}$ is given by $F^{\lambda_{n} \gamma_{n}}$. If $\mathscr{G}$ is geometric, the support of each curve in a sequence approximating $\mathscr{G}_{i}$ can be taken to be in $\operatorname{Supp} \mathscr{G}_{i}$. The flows associated to disjoint simple closed curves can easily be seen to commute.

Recall now that the pairing between differentiable functions and hamiltonian flows, defined by a symplectic structure, is continuous. We 
can therefore state:

TheOREM 7. For each element $\mathscr{G} \in \mathscr{M} \mathscr{F}$, the flow $\left(F_{t}^{\mathscr{G}}\right)_{t \in \mathbf{R}}$ is the hamiltonian flow associated to the function $\sum_{j=1}^{k} i\left(\mathscr{G}_{j}, \cdot\right)^{2}$ on $\mathscr{M} \mathscr{F}$, where $\mathscr{G}_{1}, \ldots, \mathscr{G}_{k}$ are the components of $\mathscr{G}$.

Remark that the flows $F^{\mathscr{G}}$ associated to the components $\mathscr{G}_{j}$ of $\mathscr{G}$ commute, by construction. $F_{t}^{\mathscr{G}}$ is thus obtained by composing in any $\operatorname{order} F_{t}^{\mathscr{G}_{1}}, \ldots, F_{t}^{\mathscr{G}_{k}}$.

Remarks similar to those following the statements of Theorems 4 and 5 hold. We make use of Proposition 3 and the fact that the function $i(\mathscr{G}, \cdot)$ is Lipschitz (cf. [Re]) to find coordinate charts in which the flow is the tangent flow to a vectorfield. We insist no more on these facts.

Concluding remarks. (1) geometric intersection functions are the most natural globally defined functions on $\mathscr{M} \mathscr{F}$; it would be interesting to study other kinds of functions on this space, in relation to the symplectic structure.

(2) An apparently important problem is to study what relations can there be between the symplectic structure on $\mathscr{M} \mathscr{F}$ and that on the Teichmüller space of the surface, studied by Scott Wolpert [Wo], and with more general symplectic structures, on spaces of representations of the fundamental group, studied by Bill Goldman (cf. [Go] and the bibliography there).

Acknowledgments. Helpful conversations with François Laudenbach, while this work was in progress, and with Bill Thurston, are gratefully acknowledged. In some respect, the recent works of Scott Wolpert and Bill Goldman were stimulating.

This work was done while the author was a member of the Institute for Advanced Study (Princeton).

\section{REFERENCES}

[Ab] W. Abikoff, The Real Analytic Theory of Teichmuller Space, Lecture notes in Math., 820 (1980).

[Ca] A. J. Casson, Automorphisms of Surfaces After Nielsen and Thurston, lectures from a course given at the University of Texas at Austin (1982-83) notes taken by $\mathrm{S}$. Bleiler. 
[FLP] A. Fathi, F. Laudenbach and V. Poenaru, Travaux de Thurston sur les Surfaces, Astérisque 66-67 (1979).

[Fl] W. Floyd, Incompressible surfaces in 3-manifolds: the space of boundary curves, (preprint)

[Go] W. Goldman, The symplectic nature of fundamental groups of surfaces, Adv. in Math., 54 (1984), 200-225.

[Ka] A. Katok, Invariant measures of flows on oriented surfaces, Soviet Math. Dokl., 14 (1973), 1104-1108.

[Ke] S. Kerckhoff, The Nielsen realization problem, Ann. of Math., 117 (1983), 235-265.

[Le] G. Levitt, Feuilletages des surfaces, thesis, Un. of PARIS VII, 1983.

[Ma] H. Masur, Dense geodesics in moduli space, in Riemann Surfaces and Related Topics (I. Kra and B. Maskit, eds.), Princeton University Press, 1981.

[McC] J. D. McCarthy, Subgroups of Surface Mapping Class Groups, thesis, Columbia University, 1983.

[Pa 1] A. Papadopoulos, Difféomorphismes pseudo-Anosov et automorphismes symplectiques de l'homologie, Ann. Scient. Éc. Norm. Sup., t. 15 (1982), 543-546.

[Pa 2] R_ Réseaux ferroviaires, difféomorphismes pseudo-Anosov et automorphismes symplectiques de l'homologie, Publ. Math. Orsay, $n^{\circ}$. 83-03.

[Pa 3] _ Réseaux ferroviaires et courbes simples sur une surface, C. R. Acad. Sc. Paris, 297 (1983), 565-569.

[Pa 4] _ Deux remarques sur la géométrie symplectique de l'espace des feuilletages mesurés sur une surface, to appear in Ann. Inst. Fourier.

[Pe] R. C. Penner, A Computation of the Action of the Mapping Class Groups on Isotopy Classes of Curves and Arcs in Surfaces, thesis, M.I.T., 1982.

[Re] M. Rees, An alternative approach to the ergodic theory of measured foliations on surfaces, Erg. th. and Dynam. Syst., 1 (1981), 461-488.

[Th 1] W. Thurston, The Geometry and Topology of 3-Manifolds, lecture notes, Princeton University.

[Th 2] _ Minimal stretch maps between hyperbolic surfaces, preprint, 1984.

[Wo] S. Wolpert, On the symplectic geometry of deformations of a hyperbolic surface, Ann. of Math., 117 (1983), 207-234.

Received January 16, 1985. Partially supported by National Science Foundation grant MCS-8108814(A03) and CNRS (France).

\section{UNiversite Louis PASTeur}

7, RuE ReNe DESCARTES

67084 STRASBOURG, France 


\section{PACIFIC JOURNAL OF MATHEMATICS EDITORS}

\author{
V. S. VARADARAJAN \\ (Managing Editor) \\ University of California \\ Los Angeles, CA 90024 \\ Herbert Clemens \\ University of Utah \\ Salt Lake City, UT 84112 \\ R. FINN \\ Stanford University \\ Stanford, CA 94305
}

\author{
HERMANN FLASCHKA \\ University of Arizona \\ Tucson, AZ 85721 \\ RAMESH A. GANGOLLI \\ University of Washington \\ Seattle, WA 98195 \\ VAUGHAN F. R. JONES \\ University of California \\ Berkeley, CA 94720 \\ ROBION KIRBY \\ University of California \\ Berkeley, CA 94720
}

C. C. MOORE

University of California

Berkeley, CA 94720

H. SAMELSON

Stanford University

Stanford, CA 94305

HAROLD STARK

University of California, San Diego La Jolla, CA 92093

\section{ASSOCIATE EDITORS}
R. ARENS
E. F. BECKENBACH (1906-1982)
B. H. NEUMANN
F. WOLF
K. YosHIDA

\section{SUPPORTING INSTITUTIONS}

\begin{abstract}
UNIVERSITY OF ARIZONA
UNIVERSITY OF BRITISH COLUMBIA

CALIFORNIA INSTITUTE OF TECHNOLOGY

UNIVERSITY OF CALIFORNIA

MONTANA STATE UNIVERSITY

UNIVERSITY OF NEVADA, RENO

NEW MEXICO STATE UNIVERSITY OREGON STATE UNIVERSITY
\end{abstract}

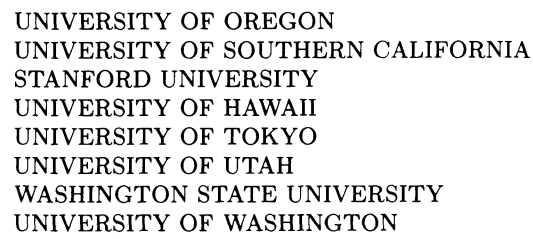

The Supporting Institutions listed above contribute to the cost of publication of this Journal, but they are not owners or publishers and have no responsibility for its content or policies.

Mathematical papers intended for publication in the Pacific Journal of Mathematics should be in typed form or offset-reproduced (not dittoed), double spaced with large margins. Please do not use built up fractions in the text of the manuscript. However, you may use them in the displayed equations. Underline Greek letters in red, German in green, and script in blue. The first paragraph must be capable of being used separately as a synopsis of the entire paper. In particular it should contain no bibliographic references. Please propose a heading for the odd numbered pages of less than 35 characters. Manuscripts, in triplicate, may be sent to any one of the editors. Please classify according to the scheme of Math. Reviews, Index to Vol. 39. Supply name and address of author to whom proofs should be sent. All other communications should be addressed to the managing editor, or Elaine Barth, University of California, Los Angeles, California 90024.

There are page-charges associated with articles appearing in the Pacific Journal of Mathematics. These charges are expected to be paid by the author's University, Government Agency or Company. If the author or authors do not have access to such Institutional support these charges are waived. Single authors will receive 50 free reprints; joint authors will receive a total of 100 free reprints. Additional copies may be obtained at cost in multiples of 50 .

The Pacific Journal of Mathematics is issued monthly as of January 1966. Regular subscription rate: $\$ 190.00$ a year (5 Vols., 10 issues). Special rate: $\$ 95.00$ a year to individual members of supporting institutions.

Subscriptions, orders for numbers issued in the last three calendar years, and changes of address should be sent to Pacific Journal of Mathematics, P.O. Box 969, Carmel Valley, CA 93924, U.S.A. Old back numbers obtainable from Kraus Periodicals Co., Route 100, Millwood, NY 10546.

The Pacific Journal of Mathematics at P.O. Box 969, Carmel Valley, CA 93924 (ISSN 0030-8730) publishes 5 volumes per year. Application to mail at Second-class postage rates is pending at Carmel Valley, California, and additional mailing offices. Postmaster: send address changes to Pacific Journal of Mathematics, P.O. Box 969, Carmel Valley, CA 93924.

PUBLISHED BY PACIFIC JOURNAL OF MATHEMATICS, A NON-PROFIT CORPORATION Copyright (C) 1986 by Pacific Journal of Mathematics 


\section{Pacific Journal of Mathematics}

Vol. 124, No. $2 \quad$ June, 1986

Philip Lee Bowers, Nonshrinkable "cell-like" decompositions of $s \ldots \ldots .257$

Aurelio Carboni and Ross Street, Order ideals in categories .......... 275

Leoni Dalla, Increasing paths on the one-skeleton of a convex compact set in

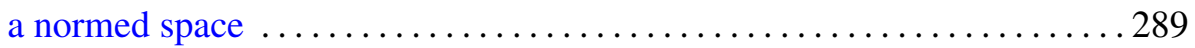

Jim Hoste, A polynomial invariant of knots and links ............... 295

Sheldon Katz, Tangents to a multiple plane curve ................... 321

Thomas George Lucas, Some results on Prüfer rings $\ldots \ldots \ldots \ldots \ldots \ldots 33$

Pham Anh Minh, Modular invariant theory and cohomology algebras of

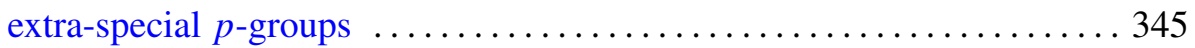

Ikuko Miyamoto, On inclusion relations for absolute Nörlund

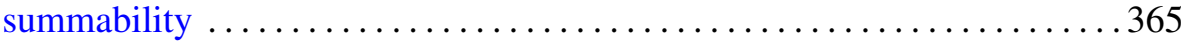

A. Papadopoulos, Geometric intersection functions and Hamiltonian flows on the space of measured foliations on a surface ............. 375

Richard Dean Resco, J. Toby Stafford and Robert Breckenridge

Warfield, Jr., Fully bounded $G$-rings $\ldots \ldots \ldots \ldots \ldots \ldots \ldots \ldots \ldots 4$

Haskell Paul Rosenthal, Functional Hilbertian sums .................417

Luen-Fai Tam, Regularity of capillary surfaces over domains with corners: borderline case ................................. 469

Hugh C. Williams, The spacing of the minima in certain cubic lattices .....483 